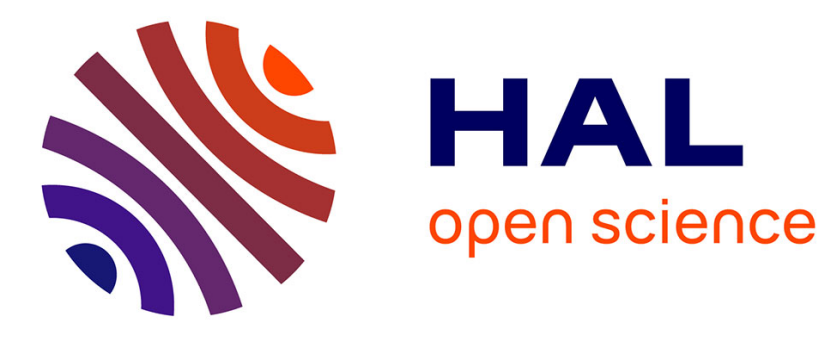

\title{
Écrire les Amériques au féminin : un regard transnational
}

Anne Reynes-Delobel, Dante Barrientos Tecun

\section{To cite this version:}

Anne Reynes-Delobel, Dante Barrientos Tecun. Écrire les Amériques au féminin : un regard transnational . Écritures dans les Amériques au féminin, 2017, 9782821895911. 10.4000/books.pup.7457 . hal-01670249

\section{HAL Id: hal-01670249 \\ https://hal.science/hal-01670249}

Submitted on 21 Mar 2018

HAL is a multi-disciplinary open access archive for the deposit and dissemination of scientific research documents, whether they are published or not. The documents may come from teaching and research institutions in France or abroad, or from public or private research centers.
L'archive ouverte pluridisciplinaire HAL, est destinée au dépôt et à la diffusion de documents scientifiques de niveau recherche, publiés ou non, émanant des établissements d'enseignement et de recherche français ou étrangers, des laboratoires publics ou privés. 


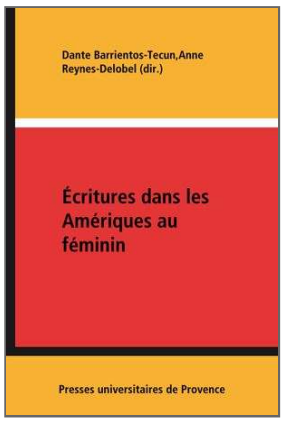

Dante Barrientos-Tecun et Anne Reynes-Delobel (dir.)

Écritures dans les Amériques au féminin

Un regard transnational

Presses universitaires de Provence

\section{Écrire les Amériques au féminin : un regard transnational}

Anne Reynes-Delobel et Dante Barrientos Tecún

DOI : 10.4000/books.pup. 7457

Éditeur: Presses universitaires de Provence

Lieu d'édition : Aix-en-Provence

Année d'édition : 2017

Date de mise en ligne : 28 septembre 2017

Collection : Textuelles

ISBN électronique : 9782821895911

\section{QboOKks}

http://books.openedition.org

Référence électronique

REYNES-DELOBEL, Anne; TECÚN, Dante Barrientos. Écrire les Amériques au féminin : un regard transnational In : Écritures dans les Amériques au féminin : Un regard transnational [en ligne]. Aix-enProvence : Presses universitaires de Provence, 2017 (généré le 24 janvier 2018). Disponible sur Internet : <http://books.openedition.org/pup/7457>. ISBN : 9782821895911. DOI : 10.4000/ books.pup. 7457 . 


\title{
Écrire les Amériques au féminin : un regard transnational
}

\author{
Anne Reynes-Delobel et Dante Barrientos Tecún
}

Nous adressons en premier lieu notre vive reconnaissance à chacun des contributeurs de cet ouvrage collectif. Nous souhaitons aussi remercier les collègues dont les noms suivent pour leur relecture attentive des articles et leurs précieuses suggestions : Françoise Buisson (Université de Pau), Juliette Bourdin (Université de Paris 8), José Garcia-Romeu (Université de Toulon), Maud Gaultier (Aix-Marseille Université), Gérard Gomez (Aix-Marseille Université), Sandra Gondouin (Université de Rouen), Diego Jarak (Université de La Rochelle), Elizabeth Lamothe (Université du Maine), Pierre Lopez (Aix-Marseille Université), Christine Lorre-Johnston (Université de Paris 3), Bénédicte Meillon (Université de Perpignan), Tabitha A. Morgan (CC Philadelphia), Nelly Rajaonarivelo (Aix-Marseille Université), Lori Saint-Martin (UQAM), Benoît Santini (Université du Littoral Côte d'Opale) et James Trombley (Université de La Rochelle). Enfin, nous exprimons notre reconnaissance à Charles Zaremba, directeur du service commun des presses d'Aix-

Marseille Université et à son équipe.

1 Les études réunies ici $^{1}$ proposent d'examiner de quelle manière les femmes écrivains " dans les Amériques » confrontent leur écriture à la représentation d'une " expérience américaine " pour en exposer les anxiétés, les failles ou les parasitages idéologiques et fantasmatiques en recourant à une subjectivité féminine travaillée par l'altérité. Ouverte à l'hétérogène et encline au déplacement et à la traversée, leur parole singulière rappelle que l'Amérique est avant tout une « invention » (O'Gorman 1961), ce qui en fait le terreau fertile de l'imaginaire, susceptible de donner naissance aux formes les plus déliées de la création artistique ou littéraire comme aux codifications les plus autoritaires du discours officiel et institutionnel. Loin de chercher à évacuer ou à lisser cette dualité, les textes analysés dans ce recueil se l'approprient pour faire jouer les oppositions et les ambiguités dans le but de révéler les récupérations symboliques, de faire resurgir les éléments expulsés des constructions historiques et mémorielles, et d'indiquer les potentialités ou les limites de la capacité d'agir (agency) du sujet. D'où l'attention portée par les auteurs de ces articles à l'analyse textuelle proprement dite, au grain de la voix du sujet de l'énonciation, à diverses figures de style (réticence, hypallage, métaphore, allégorie), au 
brouillage générique, à l'organisation rhizomatique $\mathrm{du}$ texte ou à sa texture palimpsestique, à l'acte ou au refus de nommer, ou encore au jeu poétique du vide et du blanc et à l'inscription sur le territoire virtuel de la Toile. Au fil des pages se dévoile toute la richesse d'inventivité de ces écritures d'une "américanité » plurielle, inquiète et résolument mobile dont nous allons tâcher de souligner quelques-unes des grandes lignes de force.

2 Revenons tout d'abord un instant sur la formulation employée pour introduire notre sujet. En effet, faire signe vers le domaine de la littérature des femmes n'est pas un geste neutre et appelle quelques précisions sur les présupposés critiques qui orientent notre réflexion. Les analyses recueillies dans ce volume, pour la plupart inspirées de la pensée poststructuraliste, considèrent la littérature féminine avant tout comme une pratique dans un lieu d'écriture se situant à la fois à l'intérieur et à l'extérieur de systèmes socioéconomiques, politiques, culturels, philosophiques, linguistiques et littéraires largement constitués par les hommes. Elles ne postulent donc pas l'existence d'une écriture substantiellement féminine. Elles ne s'intéressent pas non plus à des thèmes "féminins» tels la domesticité ou le monde intérieur, avec ses sentiments et ses émotions, sinon pour les intégrer à une analyse de l'espace relationnel essentielle à la pensée du fonctionnement politique moderne. Rappelons à ce propos que le changement d'approche de la dichotomie sphère publique / sphère privée dans les littératures nordet latino-américaines remonte aux débuts des années 1980. Observable chez des écrivaines comme Marilynne Robinson (Housekeeping, 1980) et Sandra Cisneros (The House on Mango Street, 1984), il a conduit au recyclage et au réagencement des tropes, des pratiques et des espaces de la domesticité, ainsi qu'à l'émergence d'environnements " alternatifs » instables et hétérogènes permettant de mieux prendre en compte l'altérité ${ }^{2}$ . De manière plus générale, il est également utile de remarquer qu'un grand nombre d'écrivaines américaines contemporaines prennent pour sujet des questions traditionnellement réservées au roman historique classique, comme l'histoire culturelle ou l'identité religieuse, pour les replacer dans un contexte transnational. Tel est le constat que dressait en 2002 l'universitaire de Cambridge et critique littéraire Gillian Beer: «Les femmes ne se limitent plus dans le choix de leurs sujets : désormais, elles n'hésitent pas à écrire sur la guerre, la famille, la communauté, les mutations sociales, le terrorisme et l'histoire. / Women have freed themselves to write more forcefully about much larger networks - wars, families, communities, national change, terrorism and history " (citée dans Showalter 2002). Cet engagement, qui témoigne plus que jamais de la volonté d'apporter une réflexion renouvelée sur le monde, s'appuie sur la notion de genre pensée comme une construction historique, sociale et mentale, et donc un outil d'analyse efficace des rapports de pouvoir. Selon cette approche, le refus d'une conception hiérarchique et trompeuse fondée sur les catégories du «masculin » et du "féminin » est le préalable indispensable à la transformation discursive et sociale des identités, des relations sociales et des relations avec le monde naturel. Le ferment anti-essentialiste de cette démarche pose inévitablement la question du lieu où la littérature croise la question politique. De fait, si de nombreuses écrivaines affirment que leurs constructions fictionnelles participent d'un geste politique ${ }^{3}$, des voix se sont élevées pour mettre en question l'efficacité d'une démarche qui, de par son hostilité à l'unité, se rend potentiellement inapte à proposer des moyens concrets d'améliorer la société et de fonder des mouvements collectifs facteurs de changement social ${ }^{4}$. S'agit-il là d'un véritable point d'achoppement ou faut-il voir dans ce constat d'une rupture avec la logique des situations 
significatives la façon dont la fiction littéraire opère des significations et se donne la possibilité de travailler la politique et d'en redessiner les contours? Cette question se trouve en filigrane au cœur des préoccupations de la plupart des analyses reprises dans cet ouvrage.

Une deuxième remarque concerne le choix d'une perspective hémisphérique. Porter le regard sur le continent américain dans son ensemble ne relève en aucun cas de la volonté de circonscrire l'Amérique ou l'américanité à un texte touchant à une essence présupposée de l'identité littéraire américaine, mais, au contraire, de prendre en compte des expériences locales, régionales ou nationales permettant tout aussi bien de repérer des phénomènes divergents que des processus comparables, des connexions, des circulations ou des interactions. C'est dans ce sens que nous avons préféré la formulation "dans les Amériques ", qui suggère une délimitation sans déterminisme, à la tournure génitive «des Amériques». Il s'agit donc d'envisager les pratiques littéraires de l'américanité dans leur pluralité sans perdre de vue les réalités économiques, culturelles et géopolitiques divergentes des États-nations américains, ainsi que le poids des héritages sur les littératures nationales. Ou, pour reprendre les propos des auteurs d'un récent recueil d'essais critiques sur les relations culturelles et littéraires entre les États-Unis et l'Amérique latine, de "ne pas abandonner le concept de nation mais d'adopter de nouvelles perspectives permettant d'envisager la nation au-delà de ses autofabulations exceptionnalistes. / Not to abandon the concept of the nation, but rather to adopt new perspectives that allow us to view the nation beyond the terms of its own exceptionnalist selfimaginings " (Levander and Levine 2008: 7). Il serait toutefois réducteur de limiter cette « déterritorialisation » des imaginaires nationaux à l'échelle hémisphérique. Comme les travaux récents des historiens de la littérature nous y invitent, nous souhaiterions les replacer dans l'écheveau des réseaux atlantiques et transpacifiques qui forment l'espace "global» dans lequel ils se sont développés au fil des siècles. Reconsidérer l'histoire littéraire nationale au prisme des dynamiques spatiales permet en effet de mettre au jour "des processus analogiques de convergence et de divergence plus complexes. / More complex, analogical processes of convergence and divergence » (Giles $2011: 23$ ). La littérature féminine dont ce livre se fait l'écho témoigne d'un désir duel d'ancrer l'écriture des Amériques dans un cadre de référence spécifique tout en déjouant l'assignation identitaire à l'origine géographique. À travers le travail de la fiction, elle reprend les données de la réalité sensible pour en modifier les cadres, les repères et les échelles, et ainsi créer un espace où se nouent de nouveaux rapports et émergent de nouveaux affects. Dans l'écart produit par ce déplacement se dessinent d'autres géographies de l'expérience américaine.

L'un des sites privilégiés d'un investissement imaginaire visant à souligner la contingence des récits nationaux, voire leur réversibilité, est la frontière. Cela n'a rien pour surprendre si l'on considère l'importance de la «Frontière » dans la construction du paysage culturel et symbolique nord-américain et latino-américain depuis l'orée du XIX siècle. Rappelons pour exemple l'ambitieux exemple de révisionnisme inversé proposé par Leslie Marmon Silko dans son roman, Almanach of the Dead (1991): en modifiant la temporalité historique et la réalité cartographique de la région frontalière MexiqueÉtats-Unis, Silko réécrit l'histoire de la région sous l'angle de la porosité transfrontalière et des hybridations raciales (espagnoles, indigènes et africaines). Aux échanges standardisés du capitalisme américano-européen, elle substitue une culture amérindienne fondée sur la transmission orale et dialogique (relayée par une écriture 
faisant la part belle à la narrativité, aux sonorités et au rythme, et invitant à l'écoute à travers l'introduction d'espaces blancs). Ainsi surgissent des héritages « oubliés " par le discours doxologique fondateur. De manière cruciale, la démarche adoptée par Silko rappelle que l'expansion territoriale des États-Unis à l'ouest du continent, au motif de leur "destinée manifeste ", a fait de la frontière non seulement ce "qu'on traverse ", mais aussi ce «qui traverse » le sujet et le langage ${ }^{5}$, mettant au jour ce qui appartient à l'altérité et à l'hétérogène. Dans cette mesure, elle peut se faire, dans l'écriture littéraire, le lieu métaphorique d'une parole poétique permettant d'évoquer avec le plus de justesse l'expérience de sujets dont l'identité est loin d'être univoque, telle la figure légendaire de la Malinche revisitée par Carmen Tafolla ou celle de la new mestiza contemporaine décrite par Gloria Anzaldúa :

Because I, a mestiza,

continually walk out of one culture

and into another,

because I am in all cultures at the same time,

alma entre dos mundos, tres, cuatro,

me zumba la cabeza con lo contradictorio.

Estoy norteada por todas las voces que me hablan

Simultáneamente. (Anzaldúa 1987 : 77)

Chez ces deux poétesses, le métissage est une dynamique qui projette le sujet de l'énonciation au-delà de l'opposition binaire (ici/là, même/différent, dedans/dehors), mais sans chercher pour autant à résoudre les contradictions ni les ambivalences. Le brouillage des langues (l'anglais et l'espagnol) génère une subjectivité labile, ouverte aux changements et aux mutations. Ici aussi, l'américanité se construit comme une forme de résistance aux interprétations élitistes (patriarcales) et aux rapports de force qui en découlent. Elle ne se veut pas tant expérience de l'« hybridité » (qui est, potentiellement, une autre forme d'essentialisme) qu'exploration de la langue dans son intimité et son étrangeté, à la charnière entre soi et l'Autre.

Chercher à approcher l'altérité par le biais de l'intimité est, de fait, un autre trait commun chez les écrivaines envisagées dans ce livre. Sur ce point, il convient toutefois de se garder des poncifs. Dans un entretien récent, la romancière et universitaire canadienne Lori Saint-Martin a ainsi pu s'agacer du fait que le terme le plus utilisé pour parler de la littérature des femmes est l'adjectif « intime », connotant le domaine privé, alors que le «masculin» continue à être considéré, y compris dans les milieux universitaires, comme étant « universel et représentatif " (Saint-Martin 2016). Devant la volonté des écrivaines contemporaines d'inscrire leur écriture dans «de plus vastes réseaux » thématiques et sémantiques, pour reprendre l'expression de Gillian Beer citée ci-dessus, on conçoit l'impatience de l'écrivaine féministe devant une forme insidieuse d'hégémonie (qu'elle désigne par le terme de " manspreading culturel »), de toute évidence dépassée. La preuve la plus flagrante a sans doute été apportée par des auteures comme Anacristina Rossi, María Rosa Lojo, Jayne Anne Phillips ou Bobbie Ann Mason qui, en adoptant directement le point de vue de combattants (hommes ou femmes) engagés dans des conflits armés, ont démontré que l'écriture de la guerre ne peut plus être considérée comme relevant de la littérature "masculine» et que les normes genrées " féminin pacifique » et « masculin guerrier » sont aussi réductrices qu'erronées. Dès lors, le constat établi par Saint-Martin signale-t-il une sorte de double bind ? Ou bien révèle-t-il une erreur d'appréciation sur la place stratégique qu'occupe l'intime dans les choix 
narratifs des écrivaines du continent? Pour tâcher de répondre à cette question, il est utile de revenir à la perspective multiscalaire mise en avant par les théoriciens de l'espace littéraire transnational afin d'établir une tension entre divers niveaux d'appréhension du réel (local, national, international ou global) permettant de mesurer la portée de l'acte narratif (individuelle ou collective) et l'importance de certains paramètres (notamment la mémoire et la corporéité).

7 Le «tournant transnational / transnational turn » (Jay 2010) pris par les études littéraires et culturelles au début des années 2000 invite en effet à envisager la production des écrivaines des Amériques à la fois dans leurs frontières nationales et hors de celles-ci, autrement dit dans un espace élargi où l'on peut relever de nouveaux points de contact et transactions. Cette perspective s'avère particulièrement intéressante pour l'analyse critique du travail des auteures binationales ou appartenant à la deuxième génération d'immigrés aux États-Unis (notamment Julia Alvarez, Edwidge Danticat, Alicia Obejas, Sandra Cisneros, Daína Chaviano, Jhumpa Lahiri, Cristina García, Dara Horn ou Gish Jen). D'une part, elle jette un nouvel éclairage sur la mise en relation de cultures locales disparates: si l'on ne s'est longtemps intéressé qu'au lien conflictuel entre ancrage territorial propre à l'exil et nostalgie du pays perdu, l'attention se porte désormais sur leur interaction dynamique. Ceci permet notamment de recontextualiser certaines œuvres dans l'espace hémisphérique transtextuel. À titre d'illustration, on peut s'attarder un instant sur le roman de Sandra Cisneros, The House on Mango Street (1984). L'œuvre de Cisneros établit en effet, à travers la référence manifeste à un roman publié par Nellie Campobello en 1931, Cartucho, une analogie perturbante entre la vie quotidienne d'un quartier latino de Chicago à l'époque contemporaine et celle d'un barrio au temps de la révolution mexicaine. En reprenant le point de vue semi-autobiographique, le type de focalisation et la structuration en vignettes adoptés par Campobello, l'écrivaine mexicoaméricaine évoque de manière subtile la façon dont un lieu accablé par la violence et la pauvreté peut devenir le site d'une action individuelle et collective à l'impact potentiellement « révolutionnaire ». Selon l'analyse récente de Geneva Gano, l'inscription intime, redéfinie à partir des coordonnées de la perspective transnationale, détermine la possibilité de penser le changement social (Gano 2015). D'autre part, la focale transnationale permet également de ne plus s'enfermer dans une pensée binaire. Ainsi, dans le roman de Cisneros, le rapprochement entre des réalités habituellement supposées distinctes, pour ne pas dire diamétralement contrastées, substitue à l'opposition « nordsud » (superpuissance états-unienne et tiers-monde ibéro-américain) la mise au jour, à l'échelle hémisphérique, de similarités troublantes et peut-être dérangeantes pour un certain lectorat. En outre, seule une analyse transnationale et globale permet de cerner les interactions de plus en plus complexes entre américanité et migration. Aux problématiques raciales, ethniques et genrées abordées par des écrivaines comme Gish Jen (Typical American, 1991), Yanitzia Canetti (Novelita rosa/Soap Opera, 1998) ou Susan Choi (American Woman, 2003) pour mettre en relief les questions liées à la citoyenneté, à l'affiliation et à l'appartenance politique auxquelles les migrantes sont confrontées dans le pays d'accueil, s'ajoutent désormais celles liées à la circulation et à l'articulation des espaces d'origine, de transit et de destination.

8 Les travaux récents des géographes ${ }^{6}$ révèlent que la migration des femmes se conçoit aujourd'hui sous la forme de mouvements de va-et-vient, de retours et de nouveaux départs, dessinant des territoires "plurilocalisés » ou «multisitués», ce qui conduit l'analyse littéraire à imaginer à son tour «des modèles transnationaux prenant en 
compte

l'espace global des déplacements continus et des connexions transcontinentales / transnational models emphasizing the global space of ongoing travel and transcontinental connection " (Friedman 2006 : 906). Dans cette mesure, si des romans comme The Agüero Sisters (1997) de l'auteure américano-cubaine Cristina García, ou, plus récemment, Americanah (2013) ou Behold the Dreamers (2016), des écrivaines d'origine africaine Chimananda Ngozi Adichi et Imbolo MBue, peuvent être considérés comme appartenant à la «littérature d'immigration », ils gagnent à être examinés sous l'angle des processus sociaux et politiques qui lient des cultures nationales hétérogènes et discontinues en réseaux. Cette approche a pour corollaire direct la remise en cause des notions d'identité linguistique unitaire et de rapport hiérarchique entre les langues : des écrivaines qui, à l'instar de Norma E. Cantú ou Edwige Danticat, pratiquent la traduction de l'anglais vers leur langue d'origine ${ }^{7}$ contribuent à la circulation des œuvres dans plusieurs traditions nationales et systèmes littéraires différents. Chez d'autres encore (Julia Alvarez, Ruth Behar), le retour physique et imaginaire vers le pays d'origine amène à une requalification de l'expérience de l'américanité à travers le vécu et la mémoire diasporiques. Cette expérience conduit également à une réinterprétation de la question identitaire envisagée en tant que notion individuelle liée à la volonté et à la conscience, par-delà des contingences strictement géographiques. Convaincus de la portée heuristique de cet imaginaire transnational, de nombreux théoriciens de la littérature ont suggéré que le terme "littérature d'immigration " soit désormais appliqué à toutes les productions composant une culture littéraire caractérisée par une vision hybride, cosmopolite et transnationale de la vie sociale (Walkowitz 2006). Aux États-Unis, certains d'entre eux n'hésitent pas à suggérer un changement paradigmatique permettant de repenser l'ensemble du système littéraire :

Plutôt que d'envisager tout ce qui relève de l'écriture littéraire (artistique, musicale, filmique) aux États-Unis à la seule lumière des textes écrits aux $\mathrm{XIX}^{\mathrm{e}}$ et $\mathrm{XX}^{\mathrm{e}}$ siècles, il serait peut-être plus pertinent de s'intéresser aux connexions avec des phénomènes qui peuvent s'observer en Amérique latine, Asie, Europe et Afrique.

What is being written (performed, composed, filmed) in the United States might most purposefully be approached less in connection to nineteenth-or twentieth-century U.S. texts and more in terms of is connections to Latin-American, Asian, European, and African phenomena. (Lauter $2010: 4$ )

Comme plusieurs études de ce volume le soulignent, un aspect important de cet imaginaire transnational réside en l'originalité d'une démarche testimoniale qui ne se contente pas de conserver la trace d'un moment-clé dans la construction d'un récit national, mais indique, comme on a pu l'observer chez Cisneros, la possibilité de le conjuguer à un autre discours fondateur pour en conjurer la logique identitaire. En transitant d'un territoire à l'autre, la mémoire est, pour ainsi dire, nettoyée de ses scories patriotiques ou idéologiques. Elle porte donc, en puissance, un imaginaire d'échanges de « bon voisinage » d'un type nouveau. En réalité, comme on le sait, l'expérience des exilées latinas aux États-Unis est souvent fort éloignée de cet idéal transnational. Ainsi qu'en témoigne de manière parodique le roman Novelita rosa/Soap Opera (1998) de Yanitzia Canetti, marginalisation, discrimination et violence urbaine sont souvent la teneur d'un quotidien traumatisant et douloureux. Ce constat, qui invite à un relativisme de bon aloi, ne saurait cependant faire oublier le rôle du travail de mémoire (souvent étayé par de patientes recherches dans les archives historiques) effectué par les écrivaines de la diaspora qui ont cherché, à l'instar de Maxine Hong Kingston ou Shirley Geok-Lin Lim, à 
explorer la subjectivité individuelle de manière critique en la mesurant à l'aune de liens familiaux, ethniques, linguistiques et culturels eux-mêmes déployés dans des espaces historiques et géographiques pluriels. D'autres auteures, comme l'écrivaine argentine María Rosa Lojo, s'attachent à fictionnaliser des figures historiques féminines de leur pays pour suggérer, entre biographie, récit de vie et saga familiale, la complexité des mécanismes d'affiliation identitaire, d'autant plus difficiles à saisir qu'ils s'inscrivent dans une relation pendulaire entre Europe et Amérique. D'autres encore, convaincues que le discours historique officiel de leur pays ne propose qu'un récit anémique du passé dans la mesure où il ignore des détails de la vie quotidienne considérés comme triviaux ou de trop peu d'importance pour avoir toute leur place dans la chronique nationale, se sont également engagées dans une poétique de la mémoire visant à remplir les blancs d'une Histoire oublieuse. C'est notamment le cas de Toni Morrison qui a tenté, dans Beloved (1987), de cerner ce qu'elle appelle la « vérité » de l'expérience de l'esclavage à travers la réécriture d'un fait divers (en l'occurrence, un infanticide commis en 1856) du point de vue de la mère meurtrière: "[The] crucial distinction for me is not the difference between fact and fiction, but the distinction between fact and truth. Because facts can exist without human intelligence, but truth cannot / Selon moi, il est crucial d'établir une distinction, non pas entre les faits et la fiction, mais entre les faits et la vérité. Si les faits peuvent exister sans le secours de l'intelligence humaine, la vérité, elle, ne le peut pas » (Morrison $2008: 72$ ). Également inspiré par le récit d'un homicide, le roman de Margaret Atwood, Alias Grace (1996), joue des tensions entre micro- et macro-histoires pour faire revivre un pan de l'Histoire du Canada du point de vue d'une main-d'œuvre ancillaire reléguée aux oubliettes de la mémoire officielle. Ajoutons brièvement que, comme celui de Morrison, le roman d'Atwood joue sur des « effets de spectralité » pour faire resurgir un passé « qui ne passe pas » sur la frontière instable entre identité individuelle et identité collective. Dans la même mouvance, citons encore la poétesse Natasha Trethewey, dont l'œuvre revisite l'histoire des prostituées métisses de la Nouvelle-Orléans au début du siècle passé. Cette plongée permet de réinscrire la tragédie "intime » (personnelle et familiale) dans un récit dont la portée collective est susceptible de bénéficier à toute une communauté.

10 Un autre constat dressé par cet ouvrage concerne la place cruciale de l'inscription corporelle dans l'imaginaire géopolitique des auteures américaines. Cet engagement du côté de la corporéité découle d'une conception de l'espace comme surface active et non passive, théorisée par la critique féministe dans le but de résister à la pensée objective et aux oppositions binaires imposées par les discours patriarcaux. En d'autres termes, en postulant "que les corps laissent leur empreinte caractéristique sur un lieu donné ", cette démarche vise à comprendre " pourquoi certains corps se sentent exclus de certains lieux, et donc à saisir la logique d'inclusion et d'exclusion à l'œuvre dans la production sociale de l'espace / Understanding [...] how bodies imprint a place with identifiable or palpable characteristics can lead to an inquiry into why some bodies feel excluded from certain places or how the social production of space operates via keeping some bodies in and some bodies out " (Reynolds 2004 : 145). Chez toutes les écrivaines convoquées dans ces pages, le recours aux tropes corporels participe d'une volonté de résister à une définition normée et fixe de la « femme », du "féminin » ou de la "féminité », relevant d'une vision androcentriste et notamment utilisée dans un but taxonomique (comme en atteste, par exemple, le célèbre ouvrage de Charles Expilly Les Femmes et les Mours du Brésil, publié en 1863). Cette résistance peut parfois, comme dans le travail de la dramaturge argentine Griselda Gambaro, prendre la forme d'une mise en abyme de la violence infligée au corps féminin 
pour inviter le lecteur (et le spectateur) à participer à un double questionnement de l'intériorisation et de l'intériorité. Chez la romancière mexicaine Guadalupe Nettel, ce processus se teinte de fantastique pour mieux mettre en relief les interactions complexes entre corps social et corps individuel, d'autant plus lorsque ce dernier devient, littéralement, une "zone occupée». Envisager le sujet féminin par le biais de la corporéité est également le moyen de déjouer le processus d'objectification à l'œuvre dans un regard distancié susceptible de transformer le sujet en symbole fantasmatique. Telle est, par exemple, la démarche adoptée par Joyce Carol Oates pour opposer, dans Blonde (2000), ses «mémoires posthumes» de Marilyn Monroe aux "mémoires imaginaires » de Norman Mailer dans of Women and Their Elegance (1980). On remarquera au passage qu'ici encore le texte fictionnel s'appuie sur le récit objectif tout en le brouillant. Dans d'autres contextes, comme ceux de l'Amérique centrale, le traitement de la corporéité chez des auteures comme Jacinta Escudos dans El hombre de la primera vez (2001) fonctionne comme une stratégie de déconstruction de la sexualité dans le régime hétérosexuel. Comme ces quelques remarques visent à le souligner, les différences épistémologiques et ontologiques dans la relation entre écriture du corps et mécanisme de production sociale de l'espace sont nombreuses, ce qui incite à faire preuve d'une grande nuance dans l'analyse des situations narratives particulières.

En suivant les études rassemblées dans cet ouvrage, il est toutefois possible de dégager certaines grandes orientations. On peut ainsi observer que, surtout chez les auteures états-uniennes, la corporéité sert à redéfinir et redéployer les modalités d'appartenance de l'individu dans le tout collectif. Le corps féminin, d'abord marqué par l'expérience de l'aliénation à soi et au reste de la communauté, est le site d'une réappropriation progressive, parfois précédée d'une phase d'« excription » sociale et/ou géographique. Au territoire physique local ou national localisable sur une carte se superpose un espace corporel genré et mobile, dont les frontières se modifient à proportion de la capacité du sujet de s'ouvrir à l'altérité de sorte à pouvoir, in fine, se porter garant de sa communauté. Ce type de trajectoire identitaire se laisse par exemple observer chez Ana Castillo, Barbara Kingsolver, Janice Ray ou Meredith Sue Willis. Chez les écrivaines écoféministes, comme Brenda Peterson ou Terri Tempest Williams, cette tendance se double d'une volonté de considérer le corps humain dans sa relation de "radicale altérité » avec l'animal afin de réinscrire les différences dans une logique d' "inclusion mutuelle" (Massumi 2014). Cette démarche s'inscrit plus largement dans une pensée "posthumaniste » visant à repenser les rapports entre l'objet et le sujet, le vivant et l'inanimé, l'humain et les autres formes d'animalité sur un mode résolument anti-identitaire (Haraway 2007). L'attention aux affects, à l'univers somatique, à l'espace et au temps prime sur celle accordée aux notions d'appartenance et d'authenticité. L'optimisme de ce monisme néo-matérialiste incarné (Gatens 1996; Braidotti 2013), dont la vocation est d'opposer une pensée "nomade » au modèle «nécropolitique » qui organise le corps social capitaliste néolibéral au plan mondial, contraste fortement avec «l'optimisme cruel » (Berlant 2011) qui caractérise l'expérience de l'américanité chez des auteures qui, à l'instar de Catherine Mavrikakis, Sharon O'Brien ou Claudia Rankine, explorent les désastres affectifs et émotionnels liés à l'attachement inconditionnel à une promesse de bonheur (communément identifiée par les termes « rêve américain » ou the good life), en dépit de la détérioration patente des conditions sociales, économiques et environnementales. Le contact frictionnel avec une réalité chaotique génère une négativité irritante, impossible à nier. Plus au sud, d'autres écrivaines exhument un continent noir de la psyché collective en établissant une autre forme de relation entre 
écriture du corps et intimité. Rosario Aguilar, Anacristina Rossi ou Vanessa Núñez Handal abordent un sujet difficile à penser, celui de la violence des femmes, notamment celle des mères responsables d'abus physiques et psychiques envers leurs filles. En abordant frontalement la question de la représentation d'une réalité dérangeante, ces auteures posent la question des rôles socialement assignés et la question de la violence comme forme d'agentivité du sujet féminin au sein du corps social. On notera enfin la prégnance d'une littérature spéculative transcontinentale (représentée par Daína Chaviano à Cuba, Ursula Le Guin aux États-Unis, Margaret Atwood au Canada, Guadalupe Nettel au Mexique et Angélica Gorodisher en Argentine) qui porte l'inscription corporelle dans des territoires imaginaires dépassant la perspective transnationale à la fois pour refléter l'étrangeté de la réalité du monde présent et indiquer les possibles scénarios de l'avenir8.

Ces grandes lignes, si elles ne résument pas, tant s'en faut, la diversité et la pluralité des représentations littéraires de l'américanité au féminin, permettent néanmoins d'établir plusieurs constatations. On observera tout d'abord la volonté des auteures de se démarquer de tout essentialisme pour réinscrire l'identité du sujet féminin dans des trajectoires mouvantes esquissant une cartographie complexe des Amériques aujourd'hui, tant sur le plan culturel et politique que sur le plan épistémologique et éthique. Envisagée sous l'angle transnational, l'américanité se dévoile dans toute la diversité de ses réalités matérielles et de ses aspirations multiples : à ses deux extrêmes, elle se révèle soit prise au piège des conditions les plus concrètes (et souvent abjectes) imposées par le modèle du néocapitalisme globalisé, soit se rêvant dans un continuum sensoriel fondé sur le contact avec l'altérité et propice à la « destinerrance » derridienne. À l'échelle hémisphérique, on relève d'importantes divergences, qui s'expliquent notamment par l'existence de contextes de violence très spécifiques dans les divers pays, au sud comme au nord, mais on peut aussi voir émerger des points de convergence transfrontaliers et parfois transhistoriques, jusque-là méconnus ou ignorés, ce qui prouve bien que la littérature est autant affaire de géopolitique que de géopoétique. À ce propos, on soulignera que le type d'inscription territoriale pratiqué par les écrivaines de l'américanité ne vise pas tant à incriminer les héritages nationaux qu'à les métisser d'autres influences, permettant au sujet de repenser sa place au sein d'une mémoire élargie. Reste à répondre à la question que nous avions laissée plus haut en suspens: quelle est la portée politique de cet imaginaire des Amériques?

13 Il ne s'agit pas ici de revenir sur l'engagement militant ou politique d'auteures qui se revendiquent ouvertement d'une littérature engagée, mais plutôt de chercher à savoir si les écritures des Amériques dont nous avons esquissé quelques-uns des principaux traits inventent aujourd'hui des catégories de déchiffrement de l'expérience commune. La question peut s'envisager dans le cadre du débat sur l'évolution de la notion d'identité dans un contexte transnational et global. En effet, si elles donnent à saisir l'expérience des Amériques dans toute son hétérogénéité sociale, culturelle et géographique, en contribuant, notamment, à mettre en lumière les contradictions induites par un modèle de mobilité néolibéral qui rend encore plus criardes les inégalités locales et régionales, cette forme de littérature ne présente aucune alternative précise. En résistant aux discours identitaires préconstruits, elle tend à la porosité des catégories d'une manière qui paraît aussi éloignée de l'affirmation que de la transgression. En établissant ce constat, nous n'entendons pas juger s'il s'agit là d'une force ou d'une faiblesse. Nous ne tendons pas non plus faire porter à ces écrivaines le poids d'une analyse sociologique qu'elles n'ont pas vocation à endosser. De plus, nous ne perdons pas de vue que les 
questions identitaires ont peu de chance d'être résolues dans des contextes sociaux où les tensions ethniques liées à l'histoire coloniale et néocoloniale sont toujours très fortes. $\mathrm{Ce}$ qui nous semble en revanche certain, c'est que la lecture et l'étude de ces écrivaines dans un contexte transnational sont un outil précieux pour comprendre les relations de pouvoir et la nécessité de repenser l'identité sur le mode relationnel de l'identification. C'est dans cet esprit que nous invitons le lecteur de cet ouvrage à aborder ces écritures au féminin qui sont autant de réinventions des Amériques pour le XXI ${ }^{\mathrm{e}}$ siècle.

ALONSO GALLO, Laura P. \& Fabio MURRIETA (eds.), 2003, Guayaba Sweet. Literatura cubana de Estados Unidos, Cádiz, Ed. Aduana Vieja.

ANZALDÚA, Gloria, 1987, "La Conciencia de le Mestiza. Towards a New Consciousness," in Borderlands/La Frontera: the New Mestiza, San Francisco, Aunt Lute, p. 77-91.

BERLANT, Lauren, 2011, Cruel Optimism, Durham, Duke University Press.

BRAIDOTTI, Rosi, 2014, "Writing as a Nomadic Subject," in Comparative Critical Studies, vol. 11, issue 2-3, Edinburgh University Press, p. 163-184.

BRAIDOTTI, Rosie, 2013, The Posthuman, Cambridge, Polity Press.

CASTRO KLAREN, Sara (ed.), 2003, Narrativa femenina en América Latina. Prácticas y

perspectivas teóricas, Madrid/Frankfurt, Iberomaricana/Vervuert.

CISNEROS, Josue David, 2014, The Border Crossed Us. Rhetorics of Borders, Citizenship, and

Latina/o Identity, Tuscaloosa, The University of Alabama Press.

DANTICAT, Evelyne, 2004, "Voices from Hispaniola," Meridians: Feminism, Race,

Transationalism, vol. 5, $\mathrm{n}^{\circ}$ 1, p. 68-91.

EPSTEIN, Barbara, 2010, « Pourquoi le poststructuralisme est une impasse pour le

féminisme ", Agone 43, p. 85-106.

EXPILLY, Charles, 1863, Les Femmes et les Mouurs du Brésil, Paris, Charlieu et Huillery.

FRIEDMAN, Susan Stanford, 2006, "Migrations, Diasporas, and Borders," in David

NICHOLLS (ed.), Introduction to Scholarship in Modern Languages and Literatures, New York,

MLA.

GANO, Geneva M., 2015, “Campobello's Cartuchos and Cisneros's Molotovs: Transborder Revolutionary Feminist Narratives," Journal of Transnational American Studies, vol. 6, issue 1 [en ligne], https://escholarship.org/uc/item/8dm6d1fz.

GATENS, Moira, 1996, Imaginary Bodies: Ethics, Power and Corporeality, London, Routledge.

GILES, Paul, 2011, The Global Remapping of American Literature, Princeton, Princeton

University Press.

GUERRA CUNNINGHAM, Lucía, 2008, Mujer y escritura. Fundamentos teóricos de la crítica feminista, Santiago, Cuarto Propio.

HARTSOCK, Nancy, 1998, The Feminist Standpoint Revisited and Other Essays, Boulder, Colorado, Westview Press, p. 205-226.

JACOBSON, Kristin J., 2010, Neodomestic American Fiction, Columbus, The Ohio State University Press.

JAY, Paul, 2010, Global Matters: The Transnational Turn in Literary Studies, Ithaca, Cornell University Press.

KINGSOLVER, Barbara, 1993, “Interview with Donna Perry," in Donna PERRY (ed.), Backtalk: Women Writers Speak Out, New Brunswick, Rutgers University, p. 143-169.

LAUTER, Paul, 2010, "Introduction," in Paul LAUTER (ed.), A Companion to American Literature and Culture, Chicester, Wiley-Blackwell.

LEVANDER, Caroline F. \& Robert S. LEVINE (eds.), 2008, "Introduction: Essays beyond the Nation," in Hemispheric American Studies, New Brunswick, Rutgers University Press, p. 1-17. 
MASSUMI, Brian, 2014, What Animals Teach Us About Politics, Durham and London, Duke University Press.

McMAHON, Marci R., 2013, Domestic Negotiations: Chicana Domesticity as a Critical Discourse, New Brunswick, Rutgers University Press.

MORRISON, Toni, 2008, What Moves at the Margin, Jackson, Mississippi, University Press of Mississippi.

O'GORMAN, Edmundo, 1958, La invención de América. Investigación acerca de la estructura histórica del nuevo mundo y del sentido de su devenir, México, Fondo de Cultura Económica. REYNOLDS, Nedra, 2004, Geographies of Writing: Inhabiting Places and Encountering Difference, Carbondale, Southern Illinois University Press.

RICHARD, Nelly, 1993, Masculino/Femenino: prácticas de la diferencia y cultura democrática, Santiago de Chile, Francisco Zegers.

RIVERO, Eliana, 1990, “(Re)Writing Sugarcane Memories: Cuban Americans and Literature," in Americas Review, 18 3-4, p. 164-182.

SAINT-MARTIN, Lori, 2016, « Prend-on la littérature des femmes au sérieux ? ", La Presse [en ligne], 8 mars, http://www.lapresse.ca/arts/livres/201603/08/01-4958501-prend-onla-litterature-des-femmes-au-serieux.php.

TAFOLLA, Carmen, 1993 [1978], "La Malinche," in Tey Diana REBOLLEDO and Eliana RIVERO, Infinite Divisions: An Anthology of Chicana Literature, Tucson, University of Arizona Press.

WALKOWITZ, Rebecca L., 2006, “The Location of Literature: The Transnational Book and the Migrant Writer," Contemporary Literature, vol. 47, issue 4, p. 527-545.

\section{NOTES}

1. Ce volume est issu de "l'axe littérature » du congrès international et transdisciplinaire de l'Institut des Amériques organisé du 4 au 6 décembre 2013 à Aix-Marseille Université en partenariat avec le Centre Aixois d'Études Romanes, avec le soutien de Sciences Po Aix, du Laboratoire d'Études et de Recherche sur le Monde Anglophone, du Laboratoire interdisciplinaire de droit des médias et des mutations sociales et du Centre d'études mexicaines et centraméricaines, et en collaboration avec le GIS Genre. Ce congrès s'était fixé pour objectif d'examiner les permanences et les mutations des rôles, des représentations et identités des femmes dans les Amériques, ainsi que la spécificité de l'aire culturelle des Amériques dans le questionnement sur le genre.

2. Plusieurs études récentes confirment ce propos (voir notamment Jacobson 2010 et McMahon 2013). Notons également l'intérêt que portent les écrivains masculins à ces espaces domestiques alternatifs, ou «néo-domestiques »: Jaime Manrique (Latin Moon in Manhattan, 1992), Richard Ford (Independence Day, 1995), Marc Z. Danielewski (House of Leaves, 2000) ou encore Cormac McCarthy (The Road, 2006) en apportent la preuve.

3. Comme le fait par exemple l'auteure écoféministe Barbara Kingsolver, qui non seulement se revendique "écrivaine politique ", mais affirme que la littérature est par essence politique, ce qui la place dans une position relativement marginale au sein de la communauté littéraire de son pays : «dans leur majorité, les écrivains états-uniens », s'étonne-t-elle, «détestent qu'on dise 
d'eux qu'ils sont des écrivains politiques. Comme si c'était une insulte. / It surprises me constantly that almost everybody else in the United States of America who writes books hates to be called a political writer. As if that demeans them " (cité dans Perry 1993 : 154). La revendication politique informe également le travail de la dramaturge Griselda Gambaro, étroitement lié à la contestation politique dans le contexte de la violence en Argentine au $\mathrm{xx}^{\mathrm{e}}$ siècle, ou encore celui des femmes écrivaines du « Roman de 30 » brésilien.

4. Certaines féministes radicales considèrent que la version du poststructuralisme adoptée par le féminisme nord-américain a principalement eu pour effet de «saper l'analyse du monde social, en remplaçant les préoccupations sociales par des préoccupations intellectuelles et esthétiques [...]. [Ce] post-modernisme a pour effet de déstabiliser les efforts tendant vers une analyse progressiste et décourage l'intérêt pour la réalité sociale " (Epstein 2010: 85). D'autres pensent que, dans la mesure où il privilégie la déconstruction sur l'action, le féminisme poststructuraliste ne propose pas d'alternative politique viable aux politiques de l'identité (Hartsock 1998 : 205-26). Par ailleurs, des auteures comme Sara Castro Klarén, Lucía Guerra Cunningham ou Nelly Richard revendiquent une spécificité de l'écriture féminine en Amérique latine du fait du contexte sociopolitique et d'une double marginalité (hiérarchie des genres et condition périphérique), remettant par là même en question la pertinence des théories féministes nord-américaines.

5. C'est le sens du célèbre slogan «We didn't cross the border, the border crossed us " repris par le mouvement de défense des droits des migrants des deux côtés de la frontière États-UnisMexique. À ce sujet, voir notamment Josue David Cisneros, The Border Crossed Us. Rhetorics of Borders, Citizenship, and Latina/o Identity, Tuscaloosa, The University of Alabama Press, 2014.

6. Voir notamment le dossier issu du congrès de l'IdA «Femmes dans les Amériques »: Amérique latine. Histoire et Mémoire, V. Baby-Collins et A. Perraudin (dir.), Les Cahiers ALHIM [en ligne], 31/2016, http://alhim.revues.org/5413.

7. De fait, il faudrait plutôt parler de retraduction, comme le souligne E. Danticat: «J'envisage toujours la traduction en termes de retraduction, car je transfère une image dans ma tête sur la page. À ceci s'ajoute le fait que la langue dans laquelle j'écris n'est pas ma langue maternelle, ce qui constitue une autre forme de traduction / I always think of the translation as a retranslation because I am transferring an image in my head onto the page. Add to this that my native language is not the one I am writing in and you also have another kind of translation " (Danticat $2004: 69$ ).

8. Au regard de la rapidité des mutations technologiques, scientifiques et médicales actuelles, on peut formuler l'hypothèse que cette forme littéraire transgenre pourrait devenir le «grand roman global » de demain.

\section{AUTEURS}

\section{ANNE REYNES-DELOBEL}

Aix-Marseille Université, LERMA EA 853, Maître de conférences, anne.reynes@univ-amu.fr Anne Reynes-Delobel est maître de conférences à Aix-Marseille Université (AMU) et membre du Laboratoire d'études et de recherche sur le monde anglophone (LERMA, EA 850). Sa recherche porte essentiellement sur le modernisme littéraire et visuel dans une perspective transnationale. Avec Mary Ann Caws, elle est l'auteure de Glorieuses modernistes : art, écriture et modernité au féminin (Presses de l'université de Liège, 2016). Elle est également présidente de la société des 
études sur Kay Boyle (affiliée à l'American Literature Association et la Society for the Studies of American Women Writers).

Anne Reynes-Delobel is a senior lecturer at Aix-Marseille University (AMU) and a member of the research center LERMA, EA 850. Her research focuses on literary and visual modernism(s) from a transnational perspective. She is coauthor, with Mary Ann Caws, of Glorieuses modernistes : art, écriture et modernité au féminin (Presses de l'université de Liège, 2016). She is currently serving as the President of the Kay Boyle Society (an ALA and SSAWW affiliate).

Anne Reynes-Delobel es maître de conférences en la Universidad Aix-Marseille (AMU) y miembro del centro de investigación LERMA, EA 850. Su trabajo de investigación se centra esencialmente sobre el modernismo literario y visual desde una perspectiva transnacional. Con Mary Ann Caws es autora de Glorieuses modernistes : art, écriture et modernité au féminin (Presses de l'université de Liège, 2016). Es además presidenta de la sociedad de estudios sobre Kay Boyle (afiliada a la American Literature Association y a la Society for the Studies of American Women Writers).

\section{DANTE BARRIENTOS TECÚN}

Aix-Marseille Université, CAER EA 854, dante.barrientos-tecun@univ-amu.fr Dante Barrientos Tecún est professeur des universités à Aix-Marseille Université et directeur adjoint du Centre aixois d'études romanes (CAER). Ses travaux de recherche se centrent en particulier sur la littérature centraméricaine. Auteur de : Un espacio cultural excluído: la situación del escritor en Guatemala (Marges 7, Perpignan, 1991), Amérique centrale : étude de la poésie contemporaine. L'Horreur et l'espoir (Paris, L'Harmattan, 1998); éditeur de Escrituras policíacas, la Historia, la Memoria. América Latina (Bologne, Astrae Editrice / CAER, 2009), Límites, fronteras e intersecciones en América Central (Cahiers d'études romanes 28, 2014); coéditeur de : Réécritures policières (Cahiers d'études romanes 25, 2012) ; México, tierra de acogida. Transculturaciones y mestizajes en el período contemporáneo (México, UNAM / CIALC-CAER, 2014), Les formes hétérogènes du roman policier (Cahiers d'études romanes 31, 2015), Amérique francophone et Amérique latine. Rencontres contemporaines (Cahiers d'études romanes 32, CAER / UNAM, 2016). Il a publié de nombreux articles en Europe et en Amérique latine sur la poésie, la narration et le théâtre d'Amérique centrale.

Dante Barrientos Tecún is a literature professor at Aix-Marseille Université and the co-director of the Centre aixois d'études romanes (CAER). His research concentrates on Central American literature. He is the autor of Un espacio cultural excluído: la situación del escritor en Guatemala (Marges 7, Perpignan, 1991) and Amérique centrale : étude de la poésie contemporaine. L'Horreur et l'espoir (Paris, L'Harmattan, 1998). He also edited Escrituras policíacas, la Historia, la Memoria. América Latina (Bologne, Astrae Editrice/CAER, 2009) and Límites, fronteras e intersecciones en América Central (Cahiers d'études romanes 28, 2014), and coedited Réécritures policières (Cahiers d'études romanes 25, 2012); México, tierra de acogida. Transculturaciones y mestizajes en el período contemporáneo (México, UNAM/CIALC-CAER, 2014); Les formes hétérogènes du roman policier ( Cahiers d'études romanes 31, 2015); and Amérique francophone et Amérique latine. Rencontres contemporaines (Cahiers d'études romanes 32, CAER/UNAM, 2016). He has also published several articles in Europe and Latin America on Central American poetry, narrativity, and drama. Dante Barrientos Tecún es professeur des universités en Aix-Marseille Université y director adjunto del Centre aixois d'études romanes (CAER). Sus trabajos de investigación se centran en particular en la literatura centroamericana. Autor de: Un espacio cultural excluído: la situación del escritor en Guatemala (Marges 7, Perpignan, 1991), Amérique centrale : étude de la poésie contemporaine. L'Horreur et l'espoir (Paris, L'Harmattan, 1998); editor de Escrituras policíacas, la Historia, la Memoria. América Latina (Bologne, Astrae Editrice / CAER, 2009), Límites, fronteras e intersecciones en América Central (Cahiers d'études romanes 28, 2014); coeditor de: Réécritures 
policières (Cahiers d'études romanes 25, 2012); México, tierra de acogida. Transculturaciones y mestizajes en el período contemporáneo (México, UNAM/CIALC-CAER, 2014), Les formes hétérogènes du roman policier (Cahiers d'études romanes 31, 2015), Amérique francophone et Amérique latine. Rencontres contemporaines (Cahiers d'études romanes 32, CAER/UNAM, 2016). Ha publicado numerosos artículos en Europa y América Latina sobre poesía, narrativa y teatro centroamericanos. 